\section{(6) OPEN ACCESS}

\title{
The Stereotactic Intracerebral Hemorrhage Underwater Blood Aspiration (SCUBA) technique for minimally invasive endoscopic intracerebral hemorrhage evacuation
}

\author{
Christopher Paul Kellner, Alexander G Chartrain, Dominic A Nistal, Jacopo Scaggiante, \\ Danny Hom, Saadi Ghatan, Joshua B Bederson, J Mocco
}

- Additional material is published online only. To view please visit the journal online (http://dx.doi.org/10.1136/ neurintsurg-2017-013719).

Department of Neurosurgery, Icahn School of Medicine at Mount Sinai, New York City, New York, USA

\section{Correspondence to}

Dr Christopher Paul Kellner, Department of Neurosurgery, Icahn School of Medicine at Mount Sinai, New York City, New York, USA; christopher. kellner@mountsinai.org

CPK and AGC contributed equally.

Received 20 December 2017 Revised 15 February 2018 Accepted 20 February 2018 Published Online First 23 March 2018

\begin{abstract}
Background Endoscopic intracerebral hemorrhage

(ICH) evacuation techniques have gained interest

as a potential therapeutic option. However, the

instrumentation and techniques employed are still being refined to optimize hemostasis and evacuation efficiency. Objective We describe the application of a specific endoscopic technique in the treatment of ICH called the Stereotactic Intracerebral Hemorrhage Underwater Blood Aspiration (SCUBA) technique. It differs from previously described minimally invasive $\mathrm{ICH}$ interventions in that it combines two separate neuroendoscopic strategies in two phases, the first under dry-field conditions and the second using a wet-field strategy.
\end{abstract}

Methods All patients who underwent endoscopic ICH evacuation with the SCUBA technique from December 2015 to September 2017 were included.

Results The SCUBA technique was performed in 47 patients. The average evacuation percentage was $88.2 \%$ (SD 20.8). Active bleeding identified to derive from a specific source was observed in 23 (48.9\%) cases. Active bleeding was addressed with irrigation alone in five cases $(10.6 \%)$ and required electrocautery in 18 cases $(38.3 \%)$. Intraoperative bleeding occurred in 3 patients $(6.4 \%)$ and postoperative bleeding occurred in a single case $(2.1 \%)$.

Conclusions The SCUBA technique provides surgeons with a defined strategy for true endoscopic hematoma evacuation. In particular, the fluid-filled cavity in SCUBA Phase 2 has the potential to provide several advantages over the traditional air-filled strategy, including clear identification and cauterization of bleeding vessels and visualization of residual clot burden. Further investigation is necessary to compare this technique to others that are currently used.

\section{INTRODUCTION}

Spontaneous ICH is a devastating and deadly form of stroke with mortality rates in excess of $40 \% .^{12}$ Trials investigating the benefit of open craniotomy and evacuation have demonstrated no discernible difference in functional outcome between the surgical and medical treatment groups. ${ }^{34}$ However, more recent trials aimed at avoiding the invasive shortcomings of the open craniotomy trials with minimally invasive strategies, such as MISTIE II and ICES, have produced promising findings. ${ }^{5-7}$
Despite this effort, current minimally invasive techniques do not permit clear visualization of residual clot burden, nor do they permit reliable visualization and cauterization of active arterial bleeding. Here, we present a novel neuroendoscopic technique called Stereotactic ICH Underwater Blood Aspiration (SCUBA), which aims to address these shortcomings with the use of a fluid-filled cavity.

\section{METHODS}

\section{Patient selection}

All patients who underwent endoscopic ICH evacuation with the SCUBA technique from December 2015 to September 2017 were included in this retrospective case series. The indications and patient eligibility criteria used include the following: hematoma volume $>20 \mathrm{cc}$, hematoma volume stability on two CT scans 6 hours apart, National Institutes of Health Stroke Scale (NIHSS) $\geq 6$, Glasgow Coma Score (GCS) $\geq 4$, and baseline modified Rankin Score $(m R S)<4$. Full inclusion and exclusion criteria can be found in online supplementary appendix 1.

\section{Data collection}

Using preoperative CT and postoperative day 1 $\mathrm{CT}$, ICH volumes were calculated using the $\mathrm{ABC} / 2$ method. ${ }^{8}$ Volume calculations were performed by a medical student (author AGC) member of the surgical team with training and close oversight from the senior authors (authors JM and CPK). Data describing the number of bleeding vessels encountered and the techniques employed to obtain hemostasis were gathered from the operative report as detailed by the operating surgeons (authors JM and CPK).

\section{Consent}

IRB study approval and patient consent for the illustrative case example were obtained.

\section{Device}

The Apollo System (Penumbra Inc., Alameda, California) was approved in 2014 by the Food and Drug Administration (FDA) for use in the evacuation of intraventricular hemorrhage and the indication has since expanded to cover ICH. It is comprised of an 


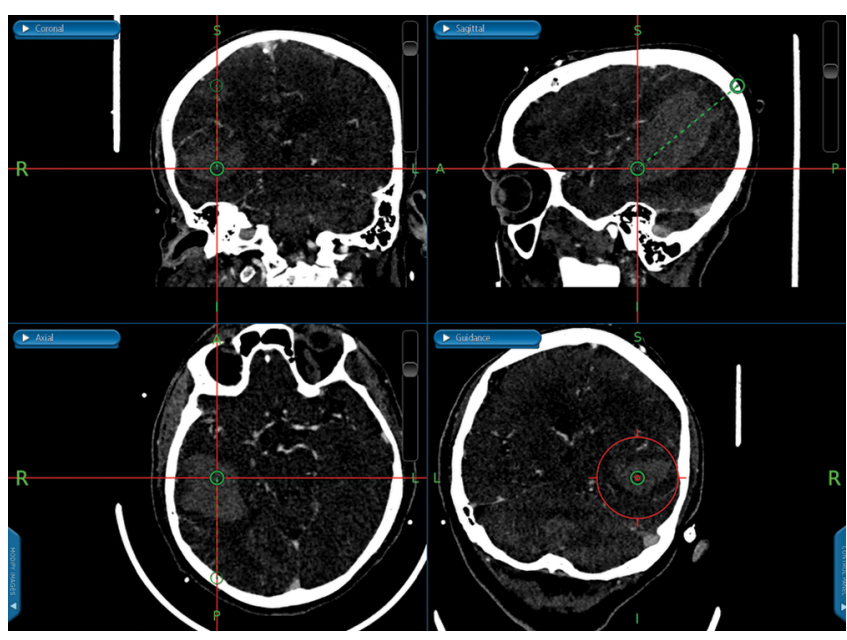

Figure 1 Preoperatively, the trajectory is planned on the stereotactic guidance system to traverse the non-eloquent brain, approach the hematoma along the long axis of the clot, and avoid vessels.

aspiration-irrigation apparatus that is connected to the Apollo wand. The Apollo wand has an outer diameter of $2.6 \mathrm{~mm}$ that is easily accommodated within the central port of an endoscope. It contains an agitator wire within its lumen that can be activated with a foot pedal to pulverize clot material. This helps to maintain patency of the slender lumen during aspiration.

\section{SCUBA (Stereotactic ICH Underwater Blood Aspiration) Positioning and planning}

Using preoperative volumetric imaging, a line is extended through the long axis of the clot to the nearest skull surface to maximize hematoma access in a single dimension while minimizing the traversed brain (figure 1). The location of prominent vasculature is taken into account when planning the entry point. The majority of supratentorial hemorrhages arise from the basal ganglia or thalamus, which are typically approached with the patient supine and the incision anterior to the coronal suture. Incisions below the hairline are accepted with care to avoid the midline and the frontal sinus. Various navigational systems are available, each with their own advantages and disadvantages. ${ }^{9}$ We prefer the Medtronic StealthStation AxiEM (Medtronic, Minneapolis, MN, USA) frameless, pinless electromagnetic system to avoid the line-of-sight limitations of alternative optical systems and the need for pinning or the attachment of a registration post, which improves the quality of the intraoperative CT.

\section{Opening}

A $3 \mathrm{~cm}$ linear, horizontal incision is made and a high-speed drill with a $5 \mathrm{~mm}$ cutting burr is used to create a $1.5 \mathrm{~cm}$ diameter craniectomy. When the trajectory is not perfectly perpendicular to the skull, extra care must be taken to drill away a cylinder in the bone along the planned trajectory to maximize mobility of the sheath and endoscope within the craniectomy. Hemostasis is attained with bone wax, gel foam, and bipolar cautery. Prior to dural incision, ultrasound may be used to confirm the hematoma size and location for comparison after evacuation. The dura is incised in a cruciate fashion and cauterized to within $1-2 \mathrm{~mm}$ of the bone edge. The insertion point is most often made in a gyrus to ensure full visibility of nearby vessels, which are carefully avoided. A pial incision is made and hemostasis is attained.
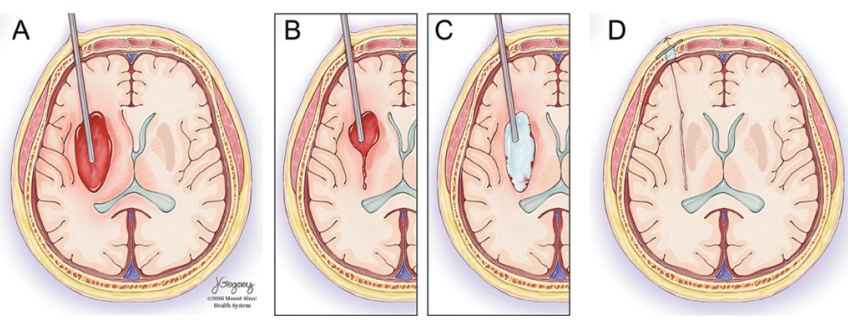

Figure 2 (A) The endoscope sheath is inserted $1.5 \mathrm{~cm}$ from the distal wall of the hematoma under stereotactic guidance. (B) Blood is aspirated until the brain closes in around the end of the sheath at which point it is retracted $1-2 \mathrm{~cm}$. (C) When the sheath retracts to the proximal wall of the hematoma cavity, saline is infused through the sheath, inflating the hematoma cavity. (D) After evacuation, the sheath is removed, and burr hole ultrasound followed by DYNA CT are performed to confirm adequate removal.

\section{Phase 1}

A 19-Fr introducer sheath is inserted along the planned trajectory with the AxiEM stylet positioned within the sheath to provide live feedback on the location of its tip. The trajectory is planned such that the tip of the sheath will rest $1.5 \mathrm{~cm}$ from the distal end of the hematoma (figure 2). At this target point, the introducer and the navigation probe are removed. The depth of the sheath is noted, marked at the level of the skin, and held in place by the assistant surgeon. The Storz (Tuttlingen, Germany) rigid endoscope is inserted. The Apollo wand is inserted in the center port and held in the opposite hand (figure 3). The suction strength on the Apollo system is set to moderate or high during this initial phase of evacuation. As described previously by Fiorella and colleagues, the suction catheter remains within the distal $1 \mathrm{~cm}$ of the sheath, aspirating liquid hematoma that presents itself in the end of the sheath (video 1$).^{10}$

After the hematoma in this region is aspirated, reduced mass effect causes the surrounding brain to collapse inward and come into view at the end of the sheath. Once this occurs, the sheath is gently pivoted at the same depth to explore laterally to the sides of the hematoma cavity (video 2). Once the operator is convinced there is no remaining hematoma at that depth, the sheath is drawn back $1.5 \mathrm{~cm}$ and the process is repeated. If, during this phase, a solid clot is encountered, the agitator wire within the Apollo wand is activated to digest large pieces. Occasionally, a formed clot is too large for the device and adheres

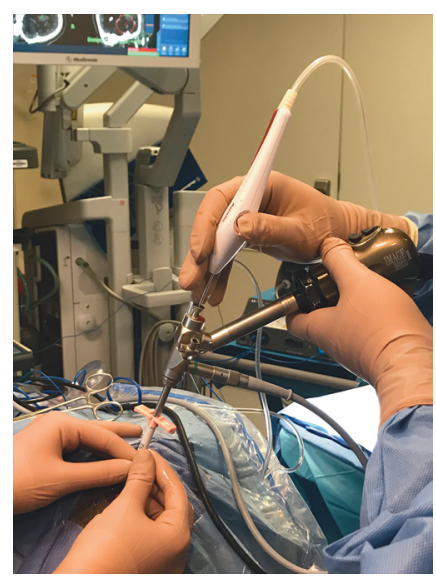

Figure 3 The endoscopic camera is held in one hand and the Apollo device in the opposite hand. The assistant surgeon holds the sheath in place and the specified depth. 


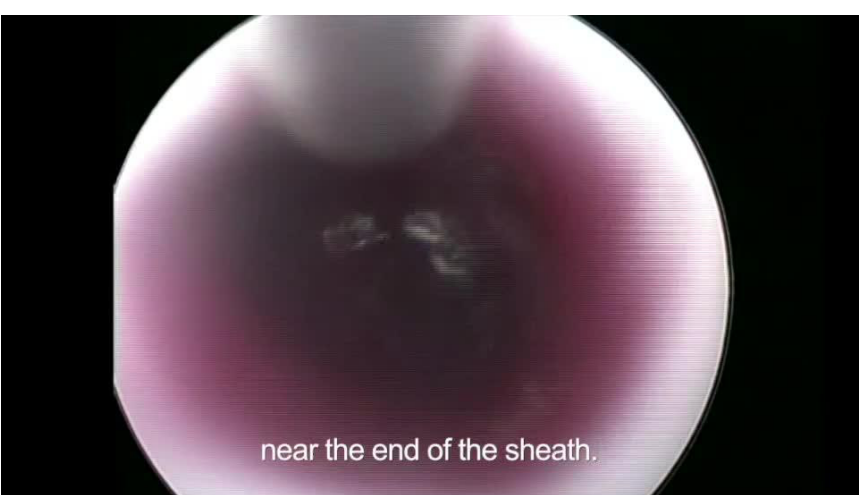

Video 1 In Phase 1 of the SCUBA technique, the APOLLO suction catheter is positioned within the endoscope sheath and advanced to its end opening. The liquid hematoma that presents to the end of the sheath is aspirated within the sheath.

to its tip. In this case, the entire endoscope and Apollo wand are removed together, with care not to dislodge the corked clot within the sheath (video 3 ).

\section{Phase 2}

After clearing all visible clots by withdrawing incrementally from the distal end of the hematoma, the signature step of the SCUBA technique is performed by infusing the cavity with normal saline from a reservoir bag suspended $\sim 25 \mathrm{~cm}$ above the level of the patient's head. This saline infuses passively through a fully opened side port while the second side port also remains fully open to permit passive egress of saline. This prevents pressurization of the cavity. With the saline freely flowing, the cavity is sufficiently expanded to permit fluid-filled exploration of the entire cavity under direct visualization. At first, blood products may obstruct visibility, but will clear after $1-5$ min with continuous irrigation. The suction is set to low $(\sim 25 \%)$ for this portion of the case to permit aspiration of blood on the wall of the cavity without damaging the nearby brain matter (video 4). If the blood does not completely clear, it may be the indication of an active bleeder. Tendrils of extravasating blood declare themselves as they diffuse from the walls into the fluid-filled cavity. Active bleeders can be addressed in several ways, including continuous irrigation, pressure from the endoscope sheath or endoscopic instrument, or cautery with an endoscopic bipolar electrode. For active bleeders that will not clear with irrigation alone, we find the bipolar cautery to be the most effective instrument due to

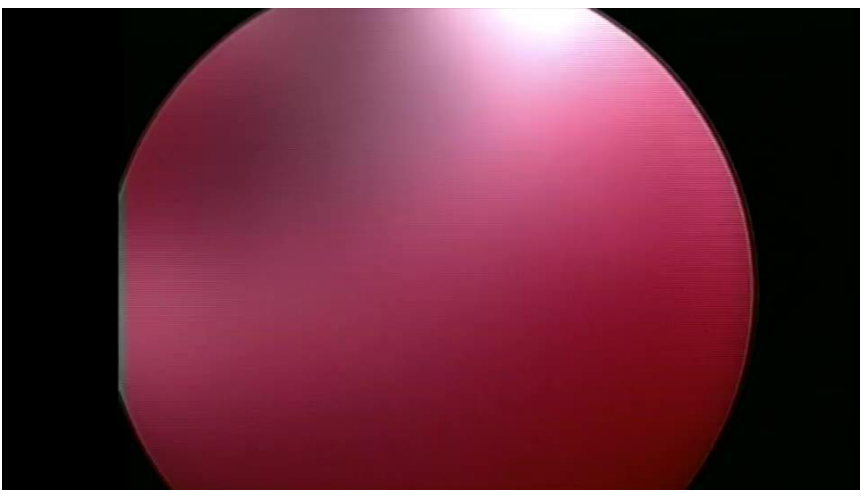

Video 2 As brain matter is seen at the end of the sheath, the operator moves the tip of the sheath laterally in both directions to evaluate the hemorrhage at the cavity wall.

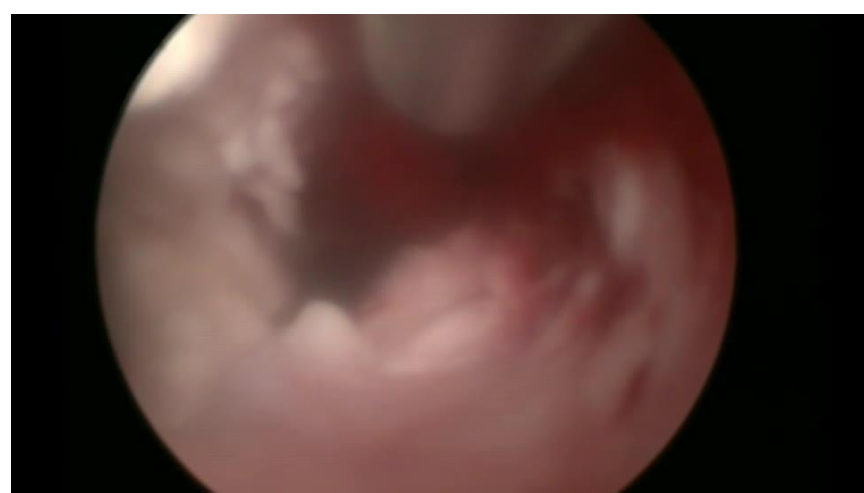

Video 3 At times, a large clot becomes stuck at the tip of the suction device and must be pulled out en masse with the endoscope to prevent the endoscope from pushing the clot off the suction device.

its rigidity and ease of navigation within view of the endoscope (video 5). After bleeders are controlled, the cavity becomes clear, permitting aspiration of smaller residual pieces of clot along the sides or in crevices of the cavity.

\section{Assessment and closure}

After aspiration of all visible clots, the sheath is removed and the burr hole ultrasound can be used to assess for residual hematoma. The ultrasound may be helpful to reassess the degree of evacuation and settle any doubt about questionable regions seen with the endoscope. Intraoperative CT is then performed prior to closure to confirm that the goal of greater than $80 \%$ of the hematoma has been evacuated. If the CT demonstrates that the residual clot volume is greater than $20 \%$ of the original volume, then a second pass is made with the introducer and sheath.

After satisfactory intra-operative imaging has been obtained, hemostatic gel foam is placed in the burr hole over the surface of the brain. A titanium plate is used to cover the craniectomy defect and is secured in place with titanium screws. The galea and subcutaneous layers are closed with 3-0 Vicryl. A 4-0 Monocryl subcuticular stitch is used to close the skin, followed by Steri-Strips.

\section{ILLUSTRATIVE CASE EXAMPLE}

The patient is an 87-year-old male with hypertension, hyperlipidemia, and coronary artery disease who presented with lethargy, left-sided hemiplegia, and left-sided neglect. On arrival at the emergency department, the patient had a GCS of 10 ,

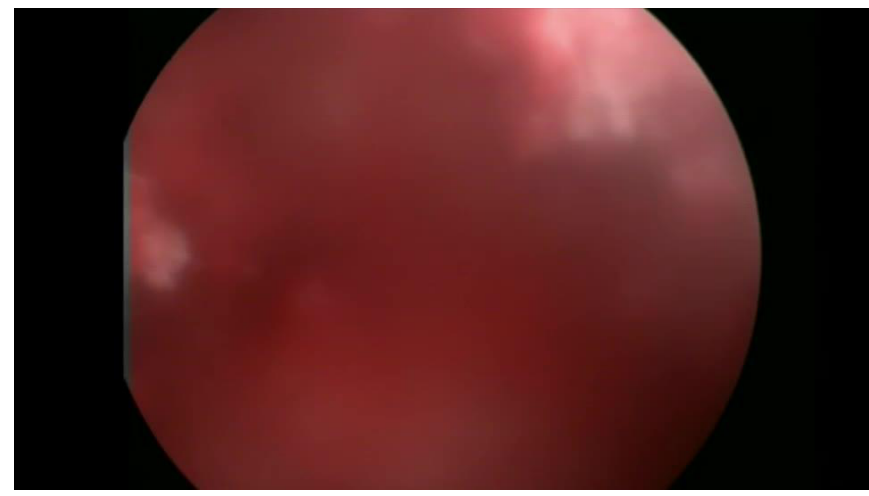

Video 4 In Phase 2 of SCUBA, normal saline freely flows into the cavity through one endoscope port and out the other. The operator evaluates the cavity wall for residual hematoma and active bleeding. 


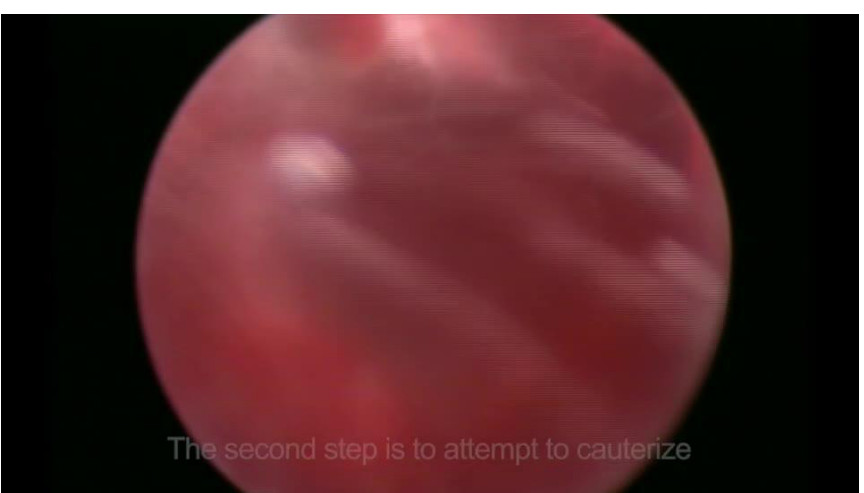

Video 5 During Phase 2 of SCUBA, if active bleeding is encountered, the first action is continuous irrigation. The second step is to attempt to cauterize the offending vessel with bipolar cautery.

NIHSS of 23, and ICH score of 4. A CT scan was performed, revealing a large, right parietal $\mathrm{ICH}$ measuring 91cc with intraventricular extension (figure $4 \mathrm{~A}-\mathrm{C}$ ). He was intubated, a right frontal EVD was placed, and he was transferred to our hospital, where he underwent minimally invasive clot evacuation 24 hours post-ictus. A trajectory was planned using stereotactic guidance and a right parietal burr hole was made. Pre-evacuation burrhole ultrasound demonstrated presence of the hematoma in the expected location (figure 5A-B).

SCUBA Phase 1 is demonstrated in the accompanying videos showing aspiration within the end of the sheath under dry-field conditions (video 1). It continues with observation of the brain closing in around the end of the sheath, lateral maneuvering of the sheath to explore the sidewalls of the cavity, and incremental retraction of the sheath (video 2). A large piece of clotted blood was found and removed by retracting the aspiration device and endoscope together (video 3). During SCUBA Phase 2 , the residual clot was aspirated from the cavity walls with gentle aspiration (video 4). Active bleeding was encountered and treated with continuous irrigation and bipolar cautery (video 5). Post-evacuation ultrasound (figure 5C-D) and intraoperative CT (figure 5E-F) confirmed $>90 \%$ evacuation. Postoperative CT demonstrated $99 \%$ evacuation with $<1 \mathrm{cc}$ residual hematoma (figure 4D-F).

The patient had a dramatic improvement to a NIHSS of 11 on postoperative day 1 and continued to improve throughout his
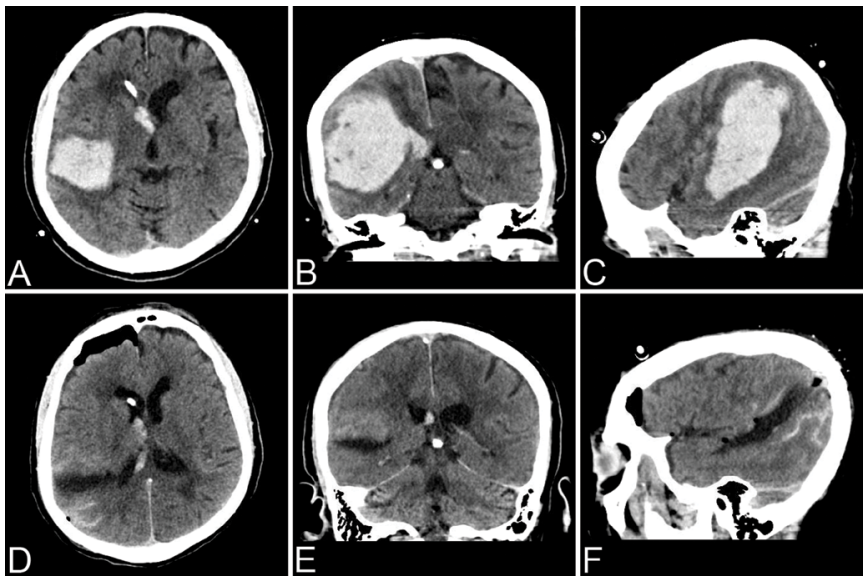

Figure 4 (A-C) Presenting head CT demonstrated a 91cc ICH with intraventricular extension. (D-F) CT performed on postoperative day 1 demonstrated $99 \%$ evacuation.
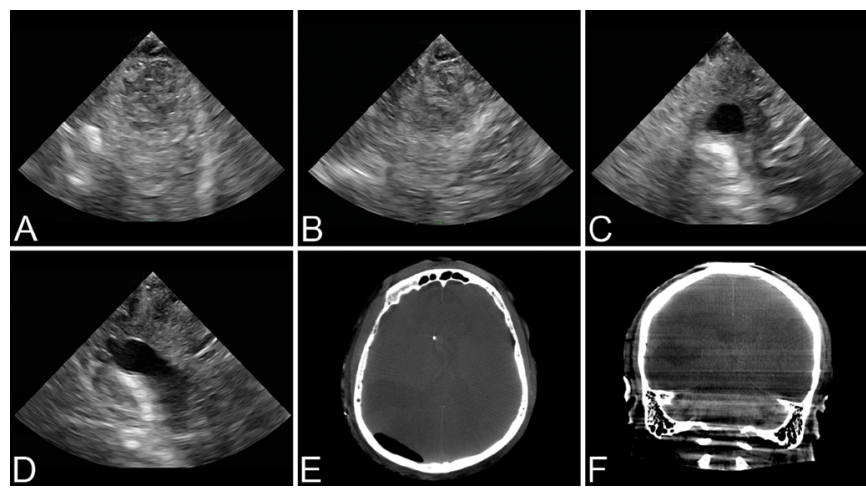

Figure 5 Pre-evacuation ultrasound demonstrated presence of the hematoma in the sagittal (A) and coronal (B) planes. Post-evacuation ultrasound demonstrated excellent clot removal with normal echogenicity of the sheath tract and resection cavity in the sagittal (C) and coronal (D) planes. Intraoperative CT confirmed $>90 \%$ evacuation in the axial (E) and coronal (F) planes.

hospitalization. Neither tracheostomy nor percutaneous gastrostomy tube placement was required during his recovery. The EVD was weaned effectively and he did not require a ventriculoperitoneal (VP) shunt prior to discharge. He was discharged on postoperative day 10 with a NIHSS of 4 . He continued to improve in acute rehabilitation and at 3-month follow-up had a NIHSS of 0 and a mRS of 1 for minor balance difficulties. At 6-month follow-up he had returned to his work as a concert pianist fulltime and was without neurological deficits (NIHSS 0 , mRS 0 ).

\section{RESULTS}

\section{Experience with the SCUBA technique}

A total of 47 patients underwent neuroendoscopic ICH evacuation via the SCUBA technique. Mean preoperative ICH volume was 42.6cc (SD 29.7) and mean postoperative ICH volume was 4.2cc (SD 6.6), amounting to an average evacuation rate of $88.2 \%$ (SD 20.8) (table 1). Active bleeding vessels were encountered during SCUBA Phase 2 in 23 cases (48.9\%) (table 2). Of these, $11(23.4 \%)$ were due to bleeding from a single vessel and $12(25.5 \%)$ were due to bleeding from multiple vessels. Bleeding was controlled by means of irrigation alone in five cases $(10.6 \%)$ and required the use of electrocautery in 18 cases (38.3\%). Intraoperative bleeding from a non-visualized source occurred in three cases $(6.4 \%)$, each of which were controlled with continuous irrigation. Postoperative re-bleeding was noted in one patient $(2.1 \%)$, on postoperative day 3 , which resulted in re-accumulation of the hematoma. The bleeding in this patient, however, was thought to derive from a superficial extracranial vessel with communication intracranially through the burr hole, as a superficial hematoma was present at the incision site.

\section{DISCUSSION}

Minimally invasive hematoma evacuation has gained popularity through multiple small, but successful, prospective trials and iterative advances in technology that address the invasive

\begin{tabular}{lc}
\hline Table 1 ICH evacuation details & \\
\hline Evacuation details & Mean (SD) \\
\hline Preoperative volume & $42.6(29.7)$ \\
Postoperative volume & $4.2(6.6)$ \\
Evacuation percentage & $88.2(20.8)$ \\
\hline
\end{tabular}


Table 2 SCUBA technique operative details

\begin{tabular}{ll}
\hline Operative details & Number (\%) \\
\hline Total patients & 47 \\
\hline Active bleeding identified & $23(48.9)$ \\
\hline Single vessel & $11(23.4)$ \\
\hline Multiple vessels & $12(25.5)$ \\
\hline Irrigation alone & $5(10.6)$ \\
\hline Electrocautery & $18(38.3)$ \\
\hline Bleeding from a non-visualized source & $3(6.4)$ \\
\hline Postoperative bleeding & $1(2.1)$ \\
\hline
\end{tabular}

drawbacks of the STICH trials technique. ${ }^{5} 61112$ The advances in minimally invasive ICH evacuation have been well described previously. ${ }^{13}$ The technique reported here is a composite of previously described minimally invasive ICH evacuation techniques that also draws on neuroendoscopic strategies developed for use in other neurosurgical procedures, including intraventricular neuroendoscopy and transsphenoidal endoscopic surgery. The novelties of the SCUBA technique include the ability to visualize residual clot burden during evacuation, and clearly identify and cauterize bleeding vessels. Further investigation is needed to compare the SCUBA technique to existing minimally invasive ICH strategies to determine its relative performance in these areas.

\section{Air versus fluid-filled evacuation}

Most authors using the Apollo System have described working within an air-filled cavity during clot evacuation. ${ }^{1014-16}$ Air-filled cavity endoscopic evacuation techniques without the Apollo System have also been described previously. ${ }^{17}$ However, Nagasaka and colleagues were the first to propose an alternative to the traditional air-filled cavity when they described the 'balanced irrigation and suction' technique. ${ }^{11}$ In this technique, when active bleeding is encountered, irrigation is increased to locate the bleeding vessel and, once found, firmly held in the suction cannula. The tip of the cannula is then electrified, which directly cauterizes the bleeding vessel. The balanced irrigation and suction technique permits the operator to search for bleeding vessels within the resection cavity and switch to an air-filled medium with aspiration before vessel ccauterization.

The SCUBA technique for attaining hemostasis of bleeding vessels within the hematoma cavity differs from Nagasaka's balanced-irrigation suction technique. With the SCUBA technique, switching to an air-filled medium prior to cauterization of the bleeding vessel is unnecessary. Instead, cauterization is completed while the cavity remains filled with irrigation fluid, which is made possible by the true endoscopic nature of the SCUBA technique. Gentle irrigation and passive fluid egress through the endoscope clears the cavity of extravasating blood from the offending vessel and maintains visualization while still submerged. A cauterization device, introduced through the central endoscope port, is placed over the bleeding vessel to cauterize the source. Performing these technical maneuvers within a fluid-filled cavity facilitates detection of persistent bleeding from these vessels in real-time and offers confirmation that hemostasis has been achieved.

The SCUBA technique is adapted from continuous irrigation strategies used in intraventricular neuroendoscopy and transsphenoidal tumor resection. ${ }^{18}{ }^{19}$ Locatelli and colleagues described a similar endoscopic 'diving technique' for transsphenoidal surgery in which continuous irrigation is used to create a fluid-filled cavity. ${ }^{18}$ The fluid-filled cavity used in the 'diving technique' permits the surgeon to evaluate the sella and intracranial skull base, wash blood from important structures, use intra-cavity pressure to quell cavernous sinus bleeding, and use the directed force of the irrigation stream to assist in tumor cleavage plane dissection. The SCUBA technique incorporates the advantages of the fluid-filled strategies used in these other forms of neuroendoscopic surgery and combines them with the previously described ICH air-filled cavity evacuation strategies.

\section{SCUBA technique considerations}

The SCUBA technique builds on previous techniques by combining stereotactic guidance, a low-profile endoscopic sheath, the Apollo System, electrocautery, and a fluid-filled cavity evacuation strategy. However, important considerations arise in response to the SCUBA technique. One concern cites that saline infusion into the hematoma cavity may cause increased intracranial pressure (ICP) or, at least, increased intracavity pressure, which may have the potential to injure viable perihematomal brain tissue. The accompanying unfavorable consequences of elevated ICP have been reported previously in the neuroendoscopic treatment of isolated IVH. ${ }^{19}$ The SCUBA technique allays concerns about this possibility by removing the majority of the hematoma and associated mass effect prior to activating any high-flow irrigation. Intracavity pressure can be monitored intraoperatively with a parenchymal ICP monitor, though this was not done for the cases described in this report.

A second point to consider is whether a greater hematoma evacuation percentage, as is the aim of the advantages offered by the SCUBA technique, is necessary for maximal improvement in functional outcome. In support of maximal evacuation, a subgroup analysis of the MISTIE II data demonstrated that increasing the evacuation percentage to greater than $70 \%$ led to improved functional outcome at 180 days compared with an evacuation percentage of $50 \%$ to $70 \%$, which itself was better than $<50 \% .^{20}$ Furthermore, animal data suggests that blood products are toxic to the surrounding brain, and implies that complete removal might be better than subtotal removal. ${ }^{21}$ Large randomized clinical trials in humans are needed to investigate this further. Until then, based on this evidence, it is reasonable to remove the maximum percentage of hematoma as is safely feasible.

The timeframe in which the evacuation procedure is performed is another important consideration. In a randomized study investigating ICH evacuation within 4 hours of symptom onset, ultra-early surgery led to higher perioperative rebleeding in comparison to surgery within 12 hours. $^{22}$ As a result, many clinicians may opt to postpone the procedure until stability of the hematoma volume has been demonstrated. However, in contrast to the craniotomy evacuation procedure used in the aforementioned study, the endoscopic evacuation technique is less invasive. Studies have suggested that minimally invasive endoscopic techniques may improve neurologic outcomes in comparison to standard craniotomy evacuation. ${ }^{23} 24$ Furthermore, ultra-early minimally invasive endoscopic evacuation has shown safety and low rebleeding rates in subsequent studies. ${ }^{25} 26$ The SCUBA technique, a true endoscopic evacuation strategy, allows for the identification of bleeding vessels and direct cauterization within a saline-filled cavity. This technique may permit safe ultra-early hematoma evacuation by increasing the likelihood of identifying a bleeding artery. Additional investigations are needed to elucidate this crucial matter and determine whether ultra-early evacuation is of potential clinical benefit to patients. 


\section{CONCLUSION}

Minimally invasive hematoma evacuation is a rapidly evolving management strategy for spontaneous ICH. The SCUBA technique represents a novel strategy utilizing the recently approved Apollo System and a fluid-filled cavity strategy for minimally invasive ICH evacuation. The fluid-filled cavity may offer advantages over air-filled and partially fluid-filled strategies that have been described previously, including clear identification and cauterization of bleeding vessels and visualization of residual clot burden, though further evaluation and comparison with existing techniques is required to determine its relative safety and efficacy.

Contributors All authors contributed to the manuscript through data collection, analysis, manuscript composition, and critical review. All authors provided final approval for publication.

Funding This research was supported in part by a grant from Arminio and Lucyna Fraga and by a grant from Mr. and Mrs. Durkovic.

Competing interests JM receives research grant support from Penumbra, Inc.

Patient consent Obtained.

Ethics approval Mount Sinai IRB.

Provenance and peer review Not commissioned; externally peer reviewed.

Open access This is an open access article distributed in accordance with the Creative Commons Attribution Non Commercial (CC BY-NC 4.0) license, which permits others to distribute, remix, adapt, build upon this work non-commercially, and license their derivative works on different terms, provided the original work is properly cited, appropriate credit is given, any changes made indicated, and the use is non-commercial. See: http://creativecommons.org/licenses/by-nc/4.0/.

\section{REFERENCES}

1 Dennis MS. Outcome after brain haemorrhage. Cerebrovasc Dis 2003;16(Suppl 1):9-13.

2 Flaherty ML, Haverbusch M, Sekar P, et al. Long-term mortality after intracerebral hemorrhage. Neurology 2006;66:1182-6.

3 Mendelow AD, Gregson BA, Fernandes HM, et al. Early surgery versus initial conservative treatment in patients with spontaneous supratentorial intracerebral haematomas in the International Surgical Trial in Intracerebral Haemorrhage (STICH): a randomised trial. Lancet 2005;365:387-97.

4 Mendelow AD, Gregson BA, Rowan EN, et al. Early surgery versus initial conservative treatment in patients with spontaneous supratentorial lobar intracerebral haematomas (STICH II): a randomised trial. Lancet 2013;382:397-408.

5 Mould WA, Carhuapoma JR, Muschelli J, et al. Minimally invasive surgery plus recombinant tissue-type plasminogen activator for intracerebral hemorrhage evacuation decreases perihematomal edema. Stroke 2013;44:627-34.

6 Vespa P, Hanley D, Betz J, et al. ICES (Intraoperative Stereotactic Computed Tomography-Guided Endoscopic Surgery) for brain hemorrhage. Stroke 2016:47:2749-55.
7 Hanley DF, Thompson RE, Muschelli J, et al. Safety and efficacy of minimally invasive surgery plus alteplase in intracerebral haemorrhage evacuation (MISTIE): a randomised, controlled, open-label, phase 2 trial. Lancet Neurol 2016;15:1228-37.

8 Kothari RU, Brott T, Broderick JP, et al. The ABCs of measuring intracerebral hemorrhage volumes. Stroke 1996;27:1304-5.

9 Chartrain AG, Kellner CP, Fargen KM, et al. A review and comparison of three neuronavigation systems for minimally invasive intracerebral hemorrhage evacuation. J Neurointerv Surg 2018;10:66-74.

10 Fiorella D, Gutman F, Woo H, et al. Minimally invasive evacuation of parenchymal and ventricular hemorrhage using the Apollo system with simultaneous neuronavigation, neuroendoscopy and active monitoring with cone beam CT. J Neurointerv Surg 2015:7:752-7.

11 Nagasaka T, Tsugeno $M$, Ikeda $H$, et al. Balanced irrigation-suction technique with a multifunctional suction cannula and its application for intraoperative hemorrhage in endoscopic evacuation of intracerebral hematomas: technical note. Neurosurgery 2009;65:E826-E827.

12 Nishihara T, Morita A, Teraoka A, et al. Endoscopy-guided removal of spontaneous intracerebral hemorrhage: comparison with computer tomography-guided stereotactic evacuation. Childs Nerv Syst 2007:23:677-83.

13 Beynon C, Schiebel P, Bösel J, et al. Minimally invasive endoscopic surgery for treatment of spontaneous intracerebral haematomas. Neurosurg Rev 2015;38:421-8.

14 Fiorella D, Arthur A, Schafer S. Minimally invasive cone beam CT-guided evacuation of parenchymal and ventricular hemorrhage using the Apollo system: proof of concept in a cadaver model. J Neurointerv Surg 2015;7:569-73.

15 Spiotta AM, Fiorella D, Vargas J, et al. Initial multicenter technical experience with the Apollo device for minimally invasive intracerebral hematoma evacuation. Neurosurgery 2015;11(Suppl 2):243-51.

16 Turner RD, Vargas J, Turk AS, et al. Novel device and technique for minimally invasive intracerebral hematoma evacuation in the same setting of a ruptured intracranial aneurysm. Neurosurgery 2015;11:43- 51

17 Dye JA, Dusick JR, Lee DJ, et al. Frontal burr hole through an eyebrow incision for image-guided endoscopic evacuation of spontaneous intracerebral hemorrhage. $J$ Neurosurg 2012;117:767-73.

18 Locatelli D, Canevari FR, Acchiardi I, et al. The endoscopic diving technique in pituitary and cranial base surgery: technical note. Neurosurgery 2010;66:E400-E401.

19 Trnovec S, Halatsch ME, Putz M, et al. Irrigation can cause prolonged intracranial pressure elevations during endoscopic treatment of intraventricular haematomas. $\mathrm{Br} J$ Neurosurg 2012;26:247-51.

20 Hanley DF. MISTIE II trial results: 365 day outcome \& cost benefit. Int Stroke Conf $2013 \mathrm{http}: / /$ braininjuryoutcomes.com/component/jdownloads/finish/64/381 ? Itemid=0 (accessed 17 Oct 2016).

21 Wu H, Wu T, Xu X, et al. Iron toxicity in mice with collagenase-induced intracerebral hemorrhage. J Cereb Blood Flow Metab 2011;31:1243-50.

22 Morgenstern LB, Demchuk AM, Kim DH, et al. Rebleeding leads to poor outcome in ultra-early craniotomy for intracerebral hemorrhage. Neurology 2001;56:1294-9.

23 Cho DY, Chen CC, Chang CS, et al. Endoscopic surgery for spontaneous basal ganglia hemorrhage: comparing endoscopic surgery, stereotactic aspiration, and craniotomy in noncomatose patients. Surg Neurol 2006;65:547-55.

24 Zhou X, Chen J, Li Q, et al. Minimally invasive surgery for spontaneous supratentorial intracerebral hemorrhage: a meta-analysis of randomized controlled trials. Stroke 2012;43:2923-30.

25 Nishihara T, Nagata K, Tanaka S, et al. Newly developed endoscopic instruments for the removal of intracerebral hematoma. Neurocrit Care 2005;2:067-74.

26 Nagasaka T, Inao S, Ikeda $\mathrm{H}$, et al. Inflation-deflation method for endoscopic evacuation of intracerebral haematoma. Acta Neurochir 2008;150:685-90. 